CASE REPORT

\title{
Di-George syndrome presenting with hypocalcaemia in adulthood: two case reports and a review
}

\author{
P S Kar, B Ogoe, R Poole, D Meeking
}

J Clin Pathol 2005;58:655-657. doi: 10.1136/icp.2004.023218

This report describes two cases of Di-George syndrome presenting with hypoparathyroidism in adulthood. The first patient presented with profound hypocalcaemia that resulted in a generalised seizure. Routine investigations revealed hypoparathyroidism. The clue to her underlying condition was the postnatal death of her young child. This case shows that $\mathrm{Di}$-George syndrome can present in adulthood with hypocalcaemia in the absence of other classic features of this condition. This has enormous implications for future family planning and may also have important health implications. The second patient, diagnosed on routine blood testing, had previously suffered with a congenital heart condition, but the syndrome was not revealed until she was of postmenopausal age. These two patients show that Di-George syndrome can present in adulthood with hypocalcaemia. This is an important observation because the condition has profound implications for health and family planning.

$\mathrm{D}$ 1-George syndrome is a dysembryonic disorder characterised by developmental abnormalities of the third and fourth pharyngeal pouches, featuring aplasia/ hypoplasia of the thymus and parathyroid glands. Other malformations include aortic arch anomalies and facial dysmorphia. Cases are often sporadic but familial cases have been described. Many affected individuals die in the neonatal period or infancy because of cardiac complications and susceptibility to infection. We report two patients presenting in adulthood. The first case is of a young adult woman presenting with a generalised seizure secondary to hypocalcaemia. The second case is an older woman presenting with hypocalcaemia on routine blood investigation. The diagnosis of Di-George syndrome has profound clinical implications for affected adults and for family planning. It is important that clinicians are aware of this condition and its presentation and clinical consequences.

\section{CASE ONE}

A 24 year old woman presented to the accident and emergency department after a witnessed seizure. While watching TV, she became rigid with hyperextension of her arms and legs. There was associated jaw clenching, tongue biting, and foaming at the mouth. She suffered a fracture to her left proximal humerus during the episode. There was no incontinence. The seizure lasted for several minutes.

There was no previous history of seizures. Her medical history included recurrent ear infections as a child and tonsillectomy/adenoidectomy. Her mother reported that she had mild learning difficulties as a child but was now fully independent, married, and in full time employment. She had no cardiac, developmental, or other medical history. Her parents were deaf, both as a result of childhood meningitis, but were otherwise fit and healthy with no past medical history. The patient had given birth to a child eight weeks previously. The child died unexpectedly 2 weeks postpartum. A necropsy showed that the baby had truncus arteriosus.

After the seizure, routine blood investigations revealed hypocalcaemia (corrected serum calcium, $1.32 \mathrm{mmol} / \mathrm{litre}$; normal range, 2.15-2.60). Subsequent investigations revealed raised serum phosphate $(1.51 \mathrm{mmol} /$ litre; normal range, $0.8-$ 1.5), raised alkaline phosphatase (118 U/litre; normal range, 30-95), and inappropriately low parathyroid hormone (0.5 pmol/litre; normal range, 0-6.4). Serum vitamin D, full blood count, serum vitamin B12, and serum folate were all normal. Tissue transglutaminase was negative. An admission electrocardiogram showed a prolongation of the QT interval (fig l).

Chromosomal analysis (fluorescence in situ hybridisation) revealed submicroscopic deletion of the 22q1l chromosome (fig 2). The same deletion was subsequently found in the patient's deceased baby.

Transthoracic echocardiography revealed no cardiac or aortic arch anomalies. A bone density scan was also normal. Immunological tests showed normal $\mathrm{T}$ cell numbers and CD4 : CD8 ratio. Chromosomal analysis of both parents showed no abnormalities.

Our patient has responded well to vitamin D treatment. She is aware of the diagnosis of Di-George syndrome and the potential risk of anomalies in future children. She is planning for her next pregnancy, and is to undergo close antenatal surveillance including chorionic villous sampling or amniocentesis.

\section{CASE 2}

A 52 year old woman presented to her general practitioner with tender cervical lymphadenopathy. Subsequent tests revealed low corrected serum calcium (2.03 mmol/litre), with raised phosphate $(1.54 \mathrm{mmol} /$ litre $)$, raised alkaline

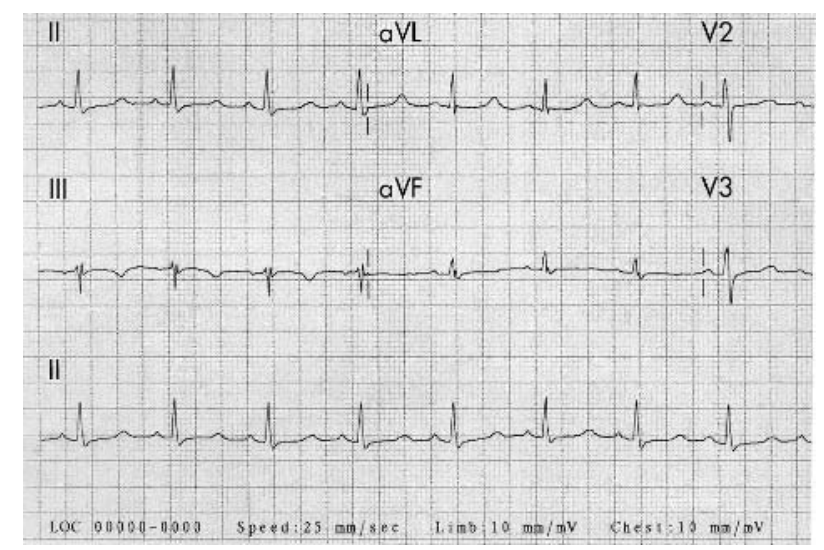

Figure 1 Admission electrocardiogram of case 1 revealing a prolonged QT interval. 


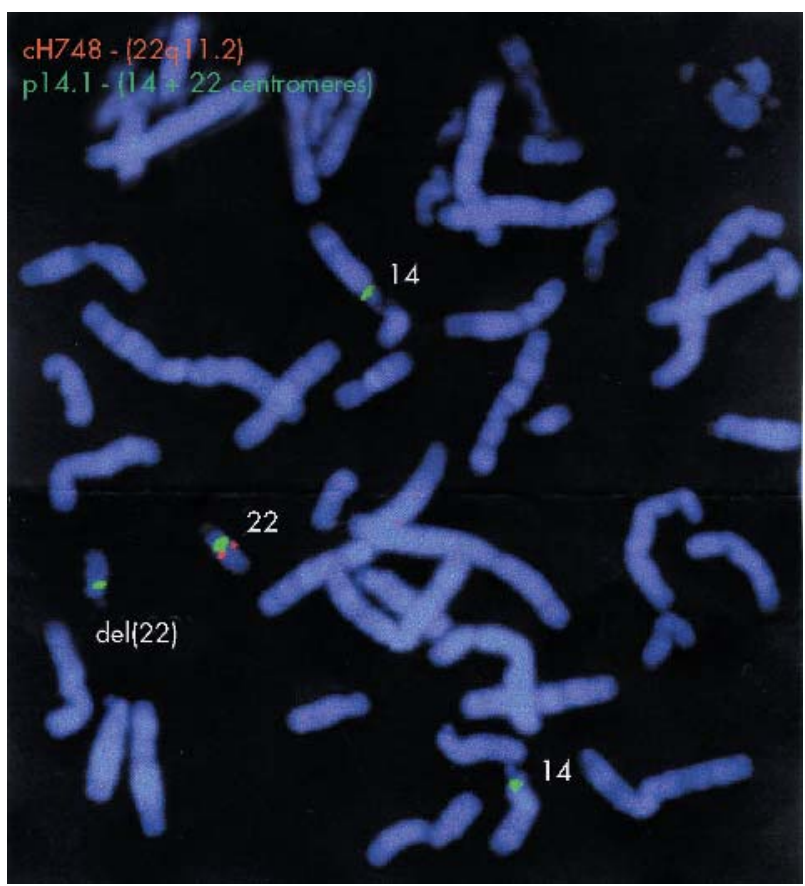

Figure 2 Chromosomal analysis using fluorescence in situ hybridisation in case 1 revealing a submicroscopic deletion of the $22 q 11$ chromosome.

phosphatase (191 U/litre), and inappropriately low parathyroid hormone ( $1.5 \mathrm{pmol} /$ litre).

The patient had a history of patent ductus arteriosus, which was receiving annual review. She had no significant family history and had conceived no children. Her past medical history was otherwise unremarkable. There was no history of intellectual impairment, recurrent infection, or renal disease. On examination, she had a round face with widely spaced eyes, low hairline, and short metacarpals. Chvostek's sign was positive. No carpo-pedal spasm was elicited.

Chromosomal analysis (fluorescence in situ hybridisation) revealed a submicroscopic deletion of the 22q1l region, consistent with Di-George syndrome.

\section{SUMMARY OF CASE REPORTS}

Our two cases show that Di-George syndrome can present with symptomatic or asymptomatic hypocalcaemia in adulthood. Our first case is unique in that the late presentation occurred in the absence of associated cardiac, facial, or severe intellectual impairment. Our second case shows that the diagnosis of Di-George syndrome may still be missed in the presence of an aortic arch anomaly and that an advanced age may be achieved without clinical manifestations.

\section{DISCUSSION}

Di-George syndrome was initially reported in $1965,{ }^{1}$ as the association of aplasia of the thymus and parathyroid glands with immunodeficiency. In 1972, Lischner ${ }^{2}$ classified DiGeorge syndrome into (a) III-IV pharyngeal pouch syndrome, (b) Di-George syndrome characterised by thymic aplasia, and (c) partial Di-George syndrome characterised by thymic hypoplasia. Most sufferers have partial Di-George syndrome.

Di-George syndrome is caused by a submicroscopic deletion of chromosome 22. It is classified along with velocardio-facial syndrome (Shprintzen syndrome) and conotruncal anomaly face syndrome as a $22 \mathrm{q} 11$ microdeletion.
The whole cohort may be referred to as the $22 \mathrm{ql} 1$ syndrome. Some have proposed the acronym "CATCH 22" (cardiac, abnormal facies, thymic hypoplasia, cleft palate, and hypocalcaemia resulting from $22 \mathrm{ql} l$ deletions) to describe the phenotype. $^{3}$

"The immune system is demonstrably affected in $80 \%$ of patients with $22 q 11$ syndrome"

Patients with Di-George syndrome can have a variety of congenital heart lesions. Kiel et al reported right aortic arch (33\%), interrupted aortic arch $(28.5 \%)$, truncus arteriosus $(28.4 \%)$, tetralogy of Fallot $(20.5 \%)$, aberrant left subclavian artery, right infundibular stenosis, and ventricular septal defect. Seventy four per cent of patients with 22qll syndrome have conotruncal malformations, ${ }^{4}$ and $69 \%$ have palatal abnormalities, including velopharyngeal incompetence, submucosal cleft palate, and cleft palate.

The immune system is demonstrably affected in $80 \%$ of patients with $22 \mathrm{ql}$ l syndrome. As a consequence of thymic hypoplasia, patients typically have reduced $\mathrm{T}$ cell numbers, although function tends to be preserved, except for the rare patients $(<0.5 \%)$ who have no $\mathrm{T}$ cells. ${ }^{6}$ The clinical consequences of the $\mathrm{T}$ cell production defect include prolonged viral infections and frequent superinfections of the upper and lower respiratory tracts. Of the patients who have a mild to moderate reduction in $\mathrm{T}$ cell production, the absolute $\mathrm{T}$ cell numbers are not predictive of infections. Approximately $25 \%$ of affected adults suffer from recurrent infections. Autoimmune disease appears to be more common in those with Di-George syndrome-both diabetes and thyroid disease have been described. Juvenile rheumatoid arthritis is the most common autoimmune disease in children with the deletion, and is found more frequently in the subset of patients with IgA deficiency. ${ }^{6}$

Hypoplastic parathyroid glands are a consequence of the malformed development of the third and fourth pharyngeal arches. In fact, neonatal hypocalcaemia is the strongest predictor of 22q11 syndrome. The hypocalcaemia generally improves over the lst year of life as the parathyroid glands hypertrophy. For those who survive, few older patients require ongoing calcium supplementation. Despite this, it is clear that hypocalcaemia can develop in older patients.

Some of the facial characteristics of Di-George syndrome are bifid uvula, high arched palate, small mouth and wide set eyes, hooded eyes, long face, malar flatness, cupped low set ears, bulbous nasal tip, and a dimpled or bifurcated nasal tip. Not all patients with 22q11 microdeletion display all, or indeed, any of these characteristics.

Renal abnormalities associated with Di-George syndrome include absent, dysplastic or multicystic kidneys, obstructive abnormalities, vesico-ureteric reflux, and nephrocalcinosis. ${ }^{3}$ Male patients may have undescended testes. Many of these abnormalities need surgical or medical intervention to prevent longterm complications, such as hypertension or

\section{Clinical manifestations/associations of Di- George syndrome}

- Hypoparathyroidism and hypocalcaemia

- Congenital heart lesions

- Immunological deficiencies

- Renal abnormalities

- Facial abnormalities

- Speech delay and learning disorders 


\section{Salient features of Di-George syndrome}

- Submicroscopic deletion of chromosome 22 (22q11 microdeletion)

- $28 \%$ of cases may have an inherited deletion

- Adult cases may present with features of hypocalcaemia

- Diagnosis in such cases has health implications and indications for genetic counselling

- Genetic testing is recommended in fetuses with cleft palate or cardiac malformation and one parent positive for the 22q11 microdeletion

- Prenatal testing widely available-diagnosis at any stage of gestation is helpful in planning for neonatal care

nephropathy, highlighting the importance of renal imaging in these patients.

Speech delay is an extremely common finding in patients with this deletion. The characteristics of speech delay are distinctive and different from the speech delay in other chromosome syndromes (such as Down syndrome). However, this careful characterisation has not translated into outcome based research on specific interventions. Learning disorders and developmental delay can affect up to $70-90 \%$ of individuals with Di-George syndrome. Another known association with this microdeletion is schizophrenia. Although there has been considerable debate about whether this is a primary association or secondary to the developmental delay, two studies suggest that the association is primary. ${ }^{6}$

The largest survey of patients with Di-George syndrome, carried out by Ryan et al, ${ }^{3}$ suggested a mortality rate of $8 \%$, with most succumbing within 6 months of birth. Sixty two per cent of surviving patients were developmentally normal or had only mild learning abnormalities, $60 \%$ were hypocalcaemic, whereas $75 \%$ had cardiological abnormalities. Thirty six per cent of patients were shown to have renal abnormalities, while $8 \%$ of male patients had undescended testes. Twenty eight per cent of the patients whose parents had been tested had inherited deletions, with a pronounced excess of maternally inherited deletions.

Of the many possible environmental explanations of the microdeletion, increased alcohol consumption during the early stages of fetal development has been proposed as an important risk factor. ${ }^{89}$

Prenatal testing for Di-George syndrome is widely available and is recommended for fetuses that have been detected as having cleft palate or a heart malformation through ultrasound, and if at least one parent tests positive for the $22 \mathrm{ql} l$ microdeletion. Diagnosis at any stage of gestation might be helpful in planning for the care of the neonate.

The available literature suggests that our two cases are rare presentations of this microdeletion. In our first case, apart from the hypocalcaemia that precipitated her seizures, there were no other hallmarks of the condition-that is,
Take home messages

- We report two rare cases of Di-George syndrome presenting with hypoparathyroidism in adulthood

- These two patients show that Di-George syndrome can present in adulthood with hypocalcaemia

- This is an important observation because the condition has profound implications for health and family planning

cardiological, developmental, neurological, or immunological abnormalities. The diagnosis was suspected because of the death of her recently born child, who was found to have the microdeletion. Previous case reports of new onset tetany or seizures caused by hypocalcaemia ${ }^{7}$ or incidental presentation $^{10}$ with previously undiagnosed disease have always revealed cardiac and/or immune abnormalities.

Thus, we suggest that primary hypocalcaemia at any age should be considered a risk factor for the deletion, and the clinical absence of the "typical" hallmarks of 22q11 microdeletion should not exclude this pathology. Our cases show that Di-George syndrome can impact in adulthood with milder effects than expected. This is important because there may be health implications and genetic counselling requirements for adult sufferers.

\section{Authors' affiliations}

P S Kar, B Ogoe, R Poole, D Meeking, Academic Department of Diabetes and Endocrinology, Queen Alexandra Hospital, Portsmouth PO6 3LY, UK

Correspondence to: Dr P Kar, Academic Department of Diabetes and Endocrinology, Queen Alexandra Hospital, Portsmouth PO6 3LY, UK; partha.kar@doctors.org.uk

Accepted for publication 19 October 2004

\section{REFERENCES}

1 DiGeorge AM. Discussion of paper by Cooper MD, Peterson RDA and Good RA: a new concept of the cellular base of immunology. J Pediatr 1965;67:907

2 Lischner HW. DiGeorge syndrome(s). J Pediatr 1972;81:1042.

3 Ryan AK, Goodship JA, Wilson DI. Spectrum of clinical features associated with interstitial chromosome 22q11 deletions: a European collaborative study. J Med Genet 1997;34:798-804.

4 Kiel EA, Drummond WH, Barrett DJ. Prevalence of T-abnormalities in infants with congenital heart disease. Am J Dis Child 1984;138:143.

5 Desmaze C, Scambler P, Prieur M. Routine diagnosis of DiGeorge syndrome by fluorescent in situ hybridization. Hum Genet 1993;90:663-5.

6 Perez E, Sullivan K. Chromosome 22q11 deletion syndrome. Curr Opin Pediatr, 2002;14:678-83.

7 Atsushi $\mathrm{H}$, Shigeto $M$, Eio K, et al. Partial DiGeorge syndrome at the age of thirty-four. Intern Med 1994;33:418-21

8 Ammann AJ, Wara DW, Cowan MJ. The DiGeorge syndrome and the fetal alcohol syndrome. Am J Dis Child 1982;136:906-8.

9 Sulik KK, Johnston MC, Daft PA, et al. Fetal alcohol syndrome and DiGeorge anomaly. Am J Med Genet Suppl 1986;2:97-112.

10 Maaswinkel-Mooii PD, Papapoulos SE, Gerritsen EJ, et al. Facial dysmorphia, parathyroid and thymic dysfunction in the father of a newborn with the DiGeorge complex. Eur J Pediatr 1989;149:179-83. 